\title{
On the infrared fluorescence of monolayer ${ }^{13} \mathrm{CO}: \mathrm{NaCl}(100)$
}

\author{
E. T. D. Boney and R. A. Marcus ${ }^{a}$ \\ Noyes Laboratory of Chemical Physics, California Institute of Technology, \\ Pasadena, California 91125-7200, USA
}

(Received 6 August 2013; accepted 17 October 2013; published online 13 November 2013)

\begin{abstract}
Computations are presented to describe and analyze the high levels of infrared laser induced vibrational excitation of a monolayer of absorbed ${ }^{13} \mathrm{CO}$ on a $\mathrm{NaCl}(100)$ surface. Extending the vibrational site-to-site surface hopping technique of Corcelli and Tully, kinetic Monte Carlo computations are used to incorporate single-quantum vibrational pooling and depooling of the ${ }^{13} \mathrm{CO}$ by phonon excitation to allow up to the $n=45$ vibrational state under different lasing conditions. Previously unpredicted pooling peaks at $n>16$ are calculated and, under the highest fluence conditions, pooling up to the $n=32$ state is found in the calculation. These results lead to the prediction of a secondary local maximum in the dispersed fluorescence of monolayer $\mathrm{CO}: \mathrm{NaCl}(100)$ under sufficiently high fluence excitation conditions. At times on the order of $\mathrm{ms}$, we recover similar behavior for both high and low fluence results. The calculations confirm that, for situations where the Debye frequency limited $n$ domain restriction approximately holds, the vibrational state population deviates from a Boltzmann population linearly in $n$, a result that we have derived earlier theoretically for a domain of $n$ restricted to one-phonon transfers. This theoretically understood term, linear in $n$, dominates the Boltzmann term and is responsible for the inversion of the population of vibrational states, $P_{n}$. (C) 2013 AIP Publishing LLC. [http://dx.doi.org/10.1063/1.4827823]
\end{abstract}

\section{INTRODUCTION}

Carbon monoxide (CO) on an $\mathrm{NaCl}(100)$ surface has been used as a model system for surface vibrational excitation since around $1990 .{ }^{1-5}$ Several low temperature experiments have been conducted on monolayers and multilayers of ${ }^{12} \mathrm{CO}$ and ${ }^{13} \mathrm{CO}$, observing overtone $(n+2 \rightarrow n)$ infrared fluorescence from monolayer ${ }^{13} \mathrm{CO}$ excitation between the 2 nd and 16 th vibrational state ${ }^{5}$ and multilayer ${ }^{13} \mathrm{CO}$ excitation reaching the 30th vibrational state. ${ }^{3,4}$ In the monolayer case, these states were inferred by application of a filter (4200-3400 $\mathrm{cm}^{-1}$ transmitted) to the total integrated overtone emission, and in the multilayer case these states were observed directly by the collection of dispersed fluorescence.

Ewing et al. ${ }^{5}$ suggested that the mechanism for this localization of vibrational energy may be the energetically favored vibrational pooling reaction. It may be recalled that pooling involves a transfer of a vibrational quantum from an adsorbed molecule on one site to another represented, for example, by $|n\rangle+|1\rangle \rightarrow|n+1\rangle+|0\rangle$ and occurs because this transfer is exothermic when the oscillators are anharmonic. This vibrational energy transfer can in principle occur with sites further removed than nearest-neighbors, but as a first approximation we focus on nearest-neighbor and single vibrational quantum exchanges.

The present formulation builds on a model of pooling and depooling rate constants given by Corcelli and Tully for this system and this surface. ${ }^{6,7}$ We also use the same Kinetic Monte Carlo (KMC) algorithm to evaluate the vibrational population evolution. 6,7 We extend their pioneering work to larger grid sizes (10 000 sites instead of 256), higher vibra-

\footnotetext{
a)Electronic mail: ram@caltech.edu
}

tional states ( $n=45$ instead of $n=15-20$ ), and to higher fluence laser conditions, including stimulated emission. Additionally, we test our previous theoretical prediction of the explicit form of the constrained vibrational population distribution function. ${ }^{8}$ In it, the $n$-domain of a pool is restricted by the maximum energy that can be dissipated by pooling exchanges exciting only single-phonons in the solid.

\section{VIBRATIONAL EXCHANGE FOR MONOLAYER CO}

In a resonant vibrational exchange, the vibrational energy of the pair of neighbors is conserved in the energy transfer, whereas in a non-resonant exchange, some of the energy excites or removes phonons from the solid. This non-resonant case provides both a ladder-climbing mechanism for vibrational excitation and a ladder-descending pathway for vibrational relaxation of the energy difference in the transition. This pooling-depooling effect is described for a vibrational state $n$ in the following equations:

$$
\begin{gathered}
|n\rangle+|m\rangle \stackrel{W_{n, m}^{p}}{\rightarrow}|n+1\rangle+|m-1\rangle, \\
|n+1\rangle+|m-1\rangle \stackrel{W_{n+1, m-1}^{d}}{\rightarrow}|n\rangle+|m\rangle,
\end{gathered}
$$

where the $W_{n, m}^{p}$ are pooling rate constants for a site in state $n$ receiving a single vibrational quantum of energy from a neighboring $\mathrm{CO}$ site initially in state $m$, and the reverse reaction is described by a depooling rate constant $W_{n+1, m-1}^{d}$.

We treat the effect of pooling on the time-evolution of $P_{n}(t)$, the probability of a single site being in the $n$th vibrational state at time $t$, where $n$ is the specific vibrational level 
(a)

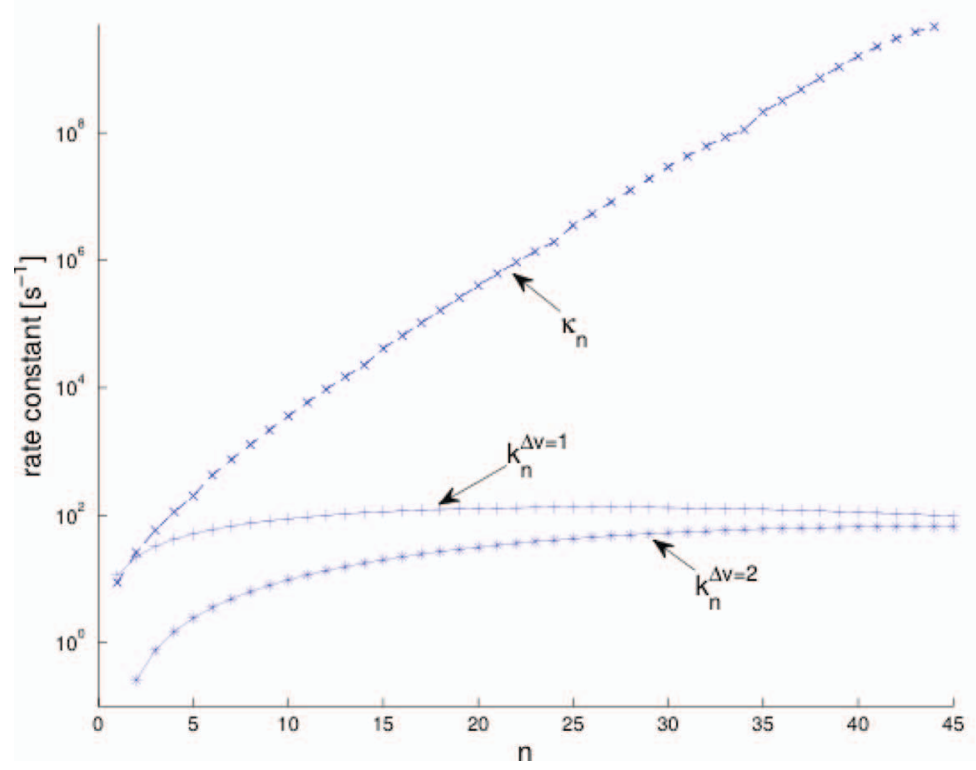

(b)

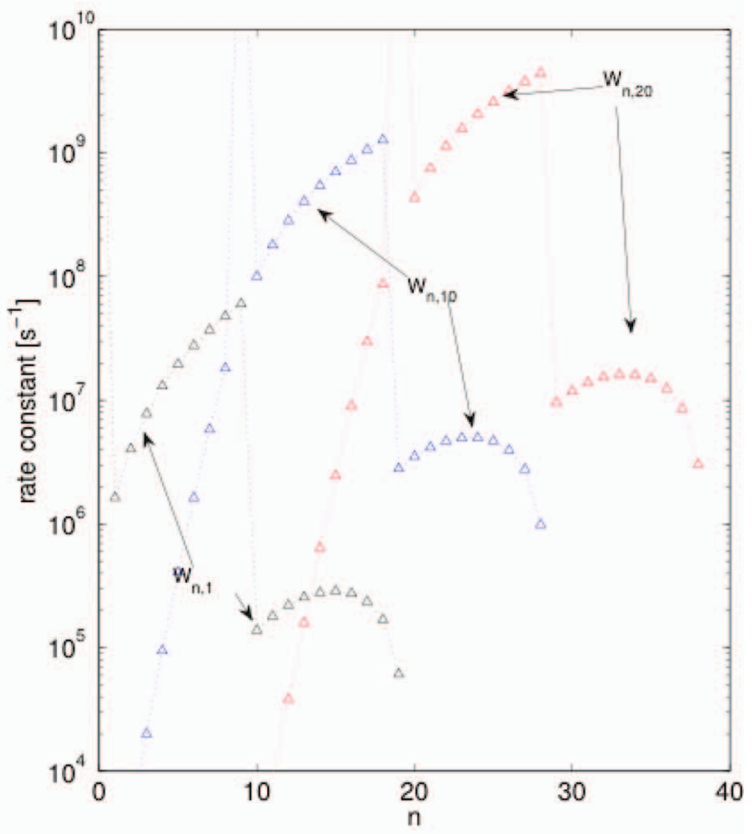

FIG. 1. Comparison of relevant rate constants: (a) first-order rate constants of relaxation $\left(\kappa_{n}\right)$, fluorescence $\left(k_{n}^{\Delta v=1}\right)$ and overtone fluorescence $\left(k_{n}^{\Delta v=2}\right)$ (b) second-order rate constants of pooling $\left(W_{n, m}^{p}\right.$ for $m=1,10$, and 20). The second-order effect of pooling to state $n$ from an $m=1$ state which requires an additional phonon to transfer to $n \geq 10$, and so $W_{10,1}^{p} \approx 10^{-3} W_{9,1}^{p}$. As second-order rate constants, the pooling and depooling rate constants should be multiplied by a conditional probability if one wishes to compare with the first-order rate constants, leading to units [ $\mathrm{s}^{-1}$ per unit conditional probability $\left.P(m \mid n)\right]$, as discussed in Appendix B.

whose time-evolution is being described. We have

$$
\sum_{n=0}^{\infty} P_{n}=1
$$

noting that, in the present model, every site is occupied and thus is in some CO vibrational state, $n=0$ and upward. Adsorption and desorption of $\mathrm{CO}$ are not considered, since the experimentally observed dissociation rate is slower than $10^{-4} \mathrm{~s}^{-1}$ at $22 \mathrm{~K},{ }^{9}$ and so should not be observed on the $20 \mathrm{~ms}$ experimental timescale, being 6 orders of magnitude slower. ${ }^{5}$ All simulations are at $22 \mathrm{~K}$, the temperature of the experiments. ${ }^{3-5}$

We define $\left(d P_{n} / d t\right)_{p d}$ as the net rate of pooling and depooling of state $n$ with nearest neighbors. The pooling and depooling terms are described in Eqs. (1) and (2) in terms of the effect on a specific vibrational state $n$,

$$
\begin{aligned}
&{\frac{d P_{n}}{d t}{ }_{p d}=} \sum_{m}\left(W_{n-1, m}^{p}\right) P_{n-1, m}+\sum_{m}\left(W_{n+1, m}^{d}\right) P_{n+1, m} \\
&-\sum_{m}\left(W_{n, m}^{p}\right) P_{n, m}-\sum_{m}\left(W_{n, m}^{d}\right) P_{n, m},
\end{aligned}
$$

where $P_{n, m}$ denotes the joint probability that there is a site in a vibrational state $n$ and that there is an adjacent site having $m$ quanta (such that $\sum_{m} P_{n, m}=4 P_{n}$, as every site has 4 neighbors). Adding relaxation steps to the above, by energy loss to the solid on which the $\mathrm{CO}$ is adsorbed, the vibrational state probability evolution of a site on a $\mathrm{CO}$ surface is

$$
{\frac{d P_{n}}{d t}}_{p d r f}={\frac{d P_{n}}{d t}}_{p-d}-\kappa_{n} P_{n}+\kappa_{n+1} P_{n+1},
$$

where the $\kappa_{n}$ denote the relaxation rate constants for transfer of energy to multiphonons in the solid. The formulae for the relevant rate constants are given in Appendix A. A comparison of the resulting rate constants of pooling, depooling, and relaxation along with fluorescence and overtone fluorescence is given for many $n$ 's in Figs. 1(a) and 1(b).

These equations are used for $1<n<45$, with a closure introduced into the equation for $n=45$ in Eq. (4) by making transfer to $P_{46}$ impossible $\left(P_{46}=0\right)$. The vibrational populations $P_{n}(t)$ can then be obtained by kinetic Monte Carlo integration ${ }^{10}$ of all possible rates, as discussed by Corcelli and Tully $^{7}$ and references cited therein, using site-to-site surface hopping methods for the energy transfer.

To allow the intensity of incoming light to be treated explicitly, we added to the KMC code terms containing the absorption coefficient $k_{a b s, l}$, the Einstein coefficient for each laser, to $\left(d P_{n} / d t\right)_{p d r f}$ in Eq. (5). We considered only singlephoton excitations from $n=0$ to the $n=1$ state,

$$
\begin{gathered}
\frac{d P_{0}}{d t}=-k_{a b s, l}\left(P_{0}-P_{1}\right)+{\frac{d P_{0}}{d t}}_{p d r f}, \\
\frac{d P_{1}}{d t}=k_{a b s, l}\left(P_{0}-P_{1}\right)+{\frac{d P_{1}}{d t}}_{p d r f},
\end{gathered}
$$

where the $\left(d P_{n} / d t\right)_{p d r f}$ are calculated as in Eq. (5) above, and $k_{a b s, \text { Mono }}=I \sigma /(\hbar \omega)$ is calculated to be $9 \times 10^{4} \mathrm{~s}^{-1}$ for monolayer laser conditions, $25 \mu \mathrm{J}$ in $5 \mu \mathrm{s}, \sigma=3 \times 10^{-17}$ $\mathrm{cm}^{2}$ molecule ${ }^{-1}$. To examine populations under lasing by higher fluence sources, we also examined excitation by the CLIO Free Electron Laser by following a single averaged 
macropulse, $k_{a b s, C L I O}=5 \times 10^{7} \mathrm{~s}^{-1}$, from $20 \mathrm{~mJ}$ in $8 \mu \mathrm{s.}{ }^{11}$ We note that, for higher fluence lasers, one must include both stimulated absorption and emission.

Given that the rate of overtone fluorescence is slower than the energy transfer from the $n$ state to multiplephonons in the solid, the total overtone fluorescence intensity at time $t, I(t)$, is given by

$$
I(t)=\frac{N_{\text {sim }}}{A} \sum_{n} k_{f}^{\Delta v=2} P_{n}(t)=\sum_{n} I_{n}(t),
$$

where $I_{n}(t)=\left(N_{\text {sim }} / A\right) k_{f}^{\Delta v=2} P_{n}(t)$ and there are $N_{\text {sim }}=10^{4}$ sites per trajectory, representing a surface area $A=1.57$ $\times 10^{-11} \mathrm{~cm}^{2}$ as in Corcelli and Tully. ${ }^{6}$ Resonant diffusion out of the illuminated spot is not considered.

The total dispersed overtone fluorescence, $S(\omega)$, is obtained by integration of the fluorescence intensity over a time $\tau_{i}$, the overtone fluorescence lines summed over all wavelengths

$$
S(\omega)=\sum_{n=2}^{45} L_{n} \int_{0}^{\tau_{i}} I_{n}(t) d t
$$

with the integrals approximated trapezoidally given $P_{n}(t)$ and $t$ from the program output up to integration time $\tau_{i}$. The times $\tau_{i}$ are chosen to represent different length fluorescence collection experiments. $L_{n}$ is the line shape, approximated for each line as a normalized Gaussian with FWHM $10 \mathrm{~cm}^{-1}$. This lineshape is preferred over the Lorentzian by a fit to the experimental dispersed overtone fluorescence emission from multilayers of $\mathrm{CO}$ on $\mathrm{NaCl}(100)$, for each overtone line, at the same temperature as the monolayer experiment. ${ }^{3,12}$ Snapshots of Eq. (9) are also given.

We study the ${ }^{13} \mathrm{CO}$ monolayer rather than a ${ }^{12} \mathrm{CO}$ system following the experimental work of Ewing et al..$^{3-5}$ and the simulation of Corcelli and Tully., ${ }^{6,7}$ This isotope was chosen for the experiments to enhance the overlap of its fundamental frequency with the ${ }^{12} \mathrm{CO}$ gas laser emission. ${ }^{13}$

Following Corcelli and Tully, ${ }^{6,7}$ the relaxation rate constants $\left(\kappa_{n}\right)$ are found by introducing a system-bath coupling scale parameter $\lambda$ which multiplies the Debye density of states as a rough estimate of the strength of coupling between surface oscillators and bath, and fixed such that the experimentally observed total overtone fluorescence lifetime $(4.3 \mathrm{~ms})$ is recovered under monolayer conditions. We find a single exponential decay matching the experimental observations, as in Fig. 2, for $\lambda=0.470$, close to the value of Corcelli and Tully $\left(\lambda_{C T}=0.522\right)$, and similar relaxation rates of $\kappa_{1}=6.7 \mathrm{~s}^{-1}$ compared to $5.7 \mathrm{~s}^{-1}$ in the previous simulation. We find different rates in the present calculation primarily because we use the experimental decay of overtone fluorescence to fix the calculated effective decay rate of overtone fluorescence, whereas the previous simulation appears to have matched the computed fundamental fluorescence decay to the experimental overtone fluorescence results. ${ }^{5-7}$

\section{KINETIC MONTE CARLO RESULTS}

We first analyze the total overtone fluorescence in Fig. 2 which has been fit via the system-bath coupling pa-

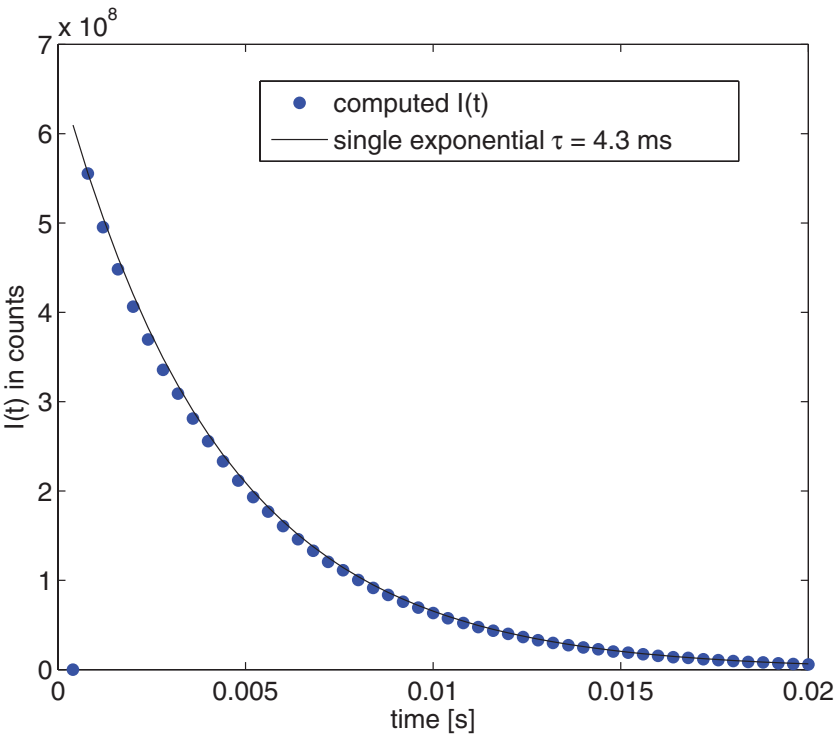

FIG. 2. The total overtone fluorescence decay (circles computed) matches experiment (solid single exponential, $I(t)=I(0) \exp (-t / \tau)$, with time constant $\tau=4.3 \mathrm{~ms})$.

rameter $\lambda$. The calculated vibrational populations at the end of each laser-on period and $1 \mathrm{~ms}$ thereafter are compared in Figs. 3 and 4, respectively. We confirm that for the monolayer, the overtone fluorescence results primarily from $n=8$ to 10 , as previously calculated. ${ }^{6,7}$

We also confirm in the computations the theoretically predicted distribution function derived in another work, ${ }^{8}$ as in Fig. 5. Shown is the deviation that is linear in $n$, a deviation from the Boltzmann distribution across the one-phonon domain restricted regime, with slope $\gamma=131$. We discuss this result further in Sec. IV.

The signal calculated in Figs. 6 and 7 is given in total photons $\mathrm{cm}^{-2}$. The signal in Fig. 8 is integrated over a short time relative to the full time of simulation. These times are effectively snapshots since the small timescale of

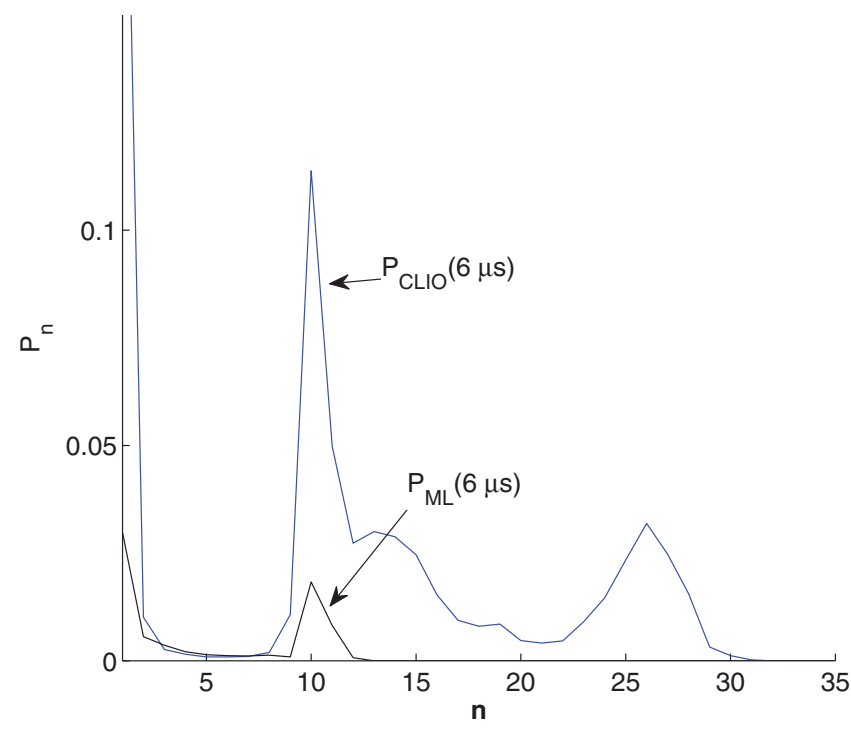

FIG. 3. The vibrational population $6 \mu$ s following beginning of pulse (near end of lasing). 


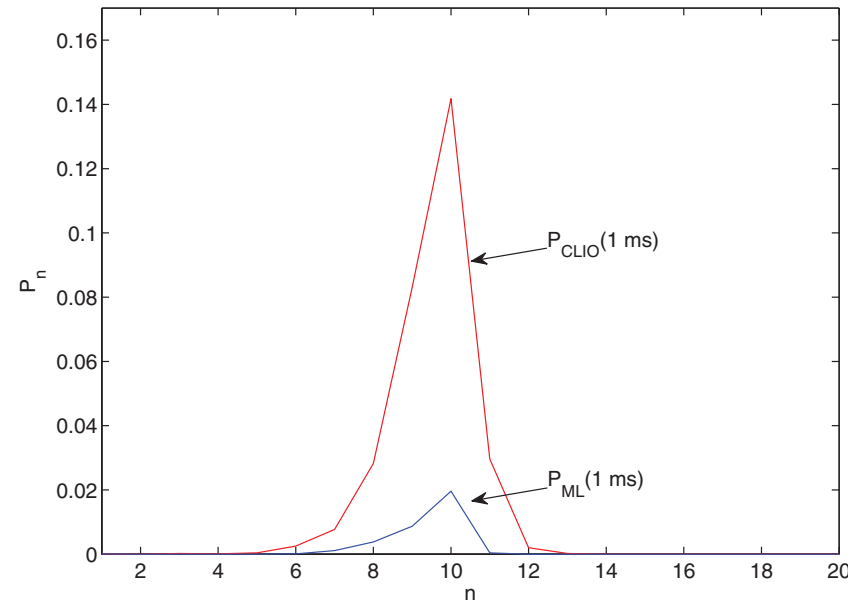

FIG. 4. The vibrational population $1 \mathrm{~ms}$ following the beginning of lasing.

the observation is ms. The timescale of the snapshot is varied between simulations, but the integration is not converged: since fluorescence is ongoing from changing populations, the timescales of integration are representative of experimental binning effects, given fixed bin sizes for collecting fluorescence ranging from $\approx 1-10 \mu \mathrm{s}$.

To understand further the evolution of the vibrational excitation of the $\mathrm{CO}$ molecules on the surface, we have examined representative snapshots from a single trajectory at the end of the first laser pulse and $1 \mathrm{~ms}$ thereafter in the high energy case in Fig. 9.

The results for the $P_{n}(t)$ are robust to grid size, since 10 trajectories on a $100 \times 100$ surface give results for the $P_{n}(t)$ that are indistinguishable from those obtained from 1 trajectory on a $300 \times 300$ grid after $1 \mathrm{~ms}$ for monolayer excitation conditions. The two sets of trajectories have roughly the same

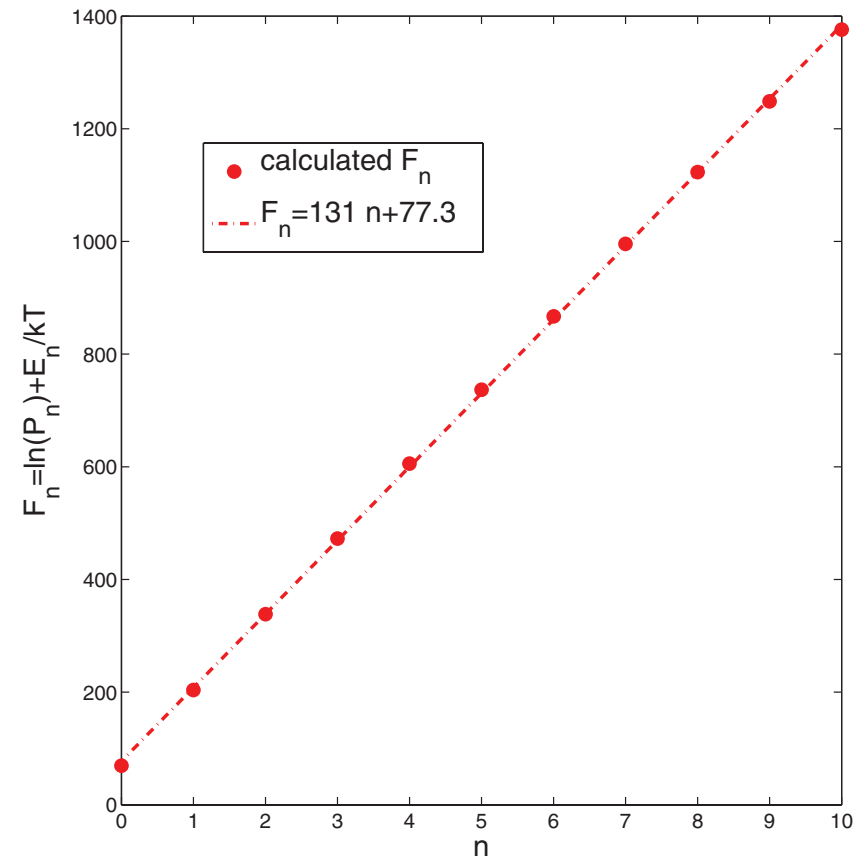

FIG. 5. The fit of the computed results to the theory-based expression permits the evaluation of statistical parameter $\gamma=131$, from the slope of the linear fit on the restricted $n$ domain. A typical result for $P_{n}$ at the conclusion of monolayer excitation is displayed, although this $\gamma$ is representative of those where the restricted $n$-domain assumption holds.

implied maximum resolution in $P_{n}(t)$, namely, $N_{\text {traj }}^{-1} N_{\text {sites }}^{-1}$ $=10^{-5}$ vs. $(1 / 9) \times 10^{-4}$. The results are also robust with respect to variation of the resonant transfer rate constant, which is reduced by three orders of magnitude to realize similar order gains in computational time. Increasing the resonant rate constant by two orders of magnitude showed no noticeable change in the results for the $P_{n}$ distribution obtained (a)

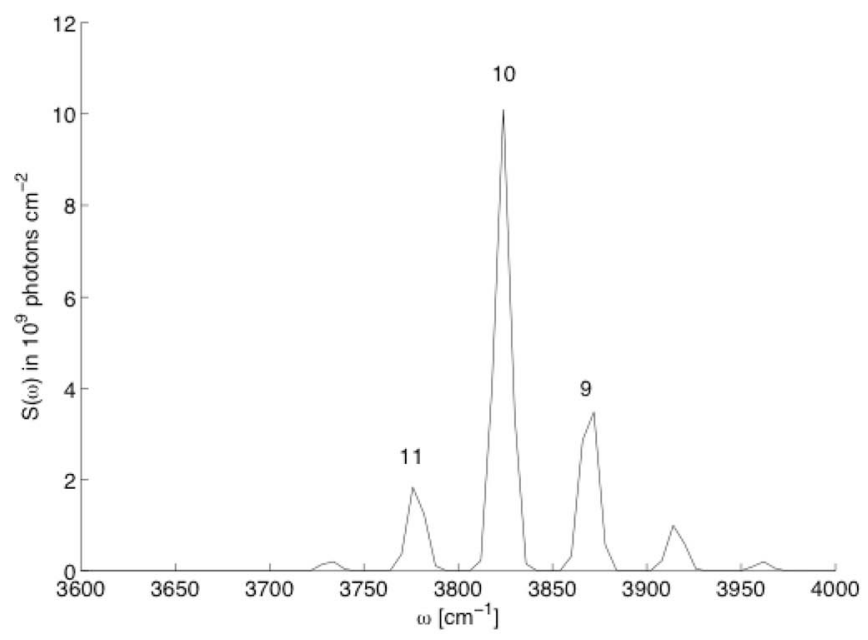

(b)

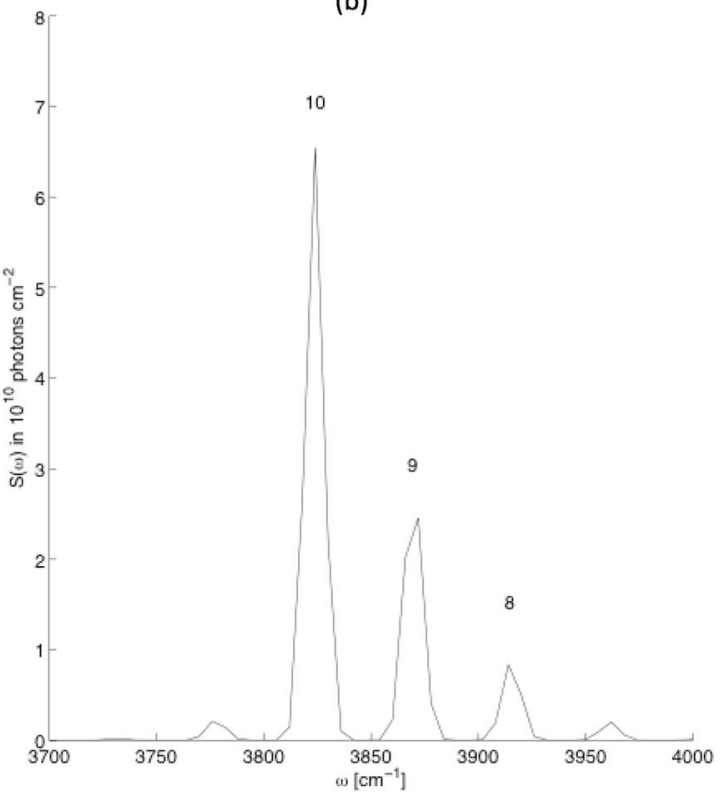

FIG. 6. The theoretical dispersed fluorescence (assuming perfect collection efficiency) for a monolayer under the experimental monolayer lasing conditions $\left(k_{a b s}=9 \times 10^{4} \mathrm{~s}^{-1}\right)$, integrated over a (a) $1 \mathrm{~ms}$ and (b) $20 \mathrm{~ms}$ period after the beginning of lasing. ${ }^{5}$ The temporal integration was calculated trapezoidally from $P_{n}(t)$ at 79 time points spread equally over the length of calculation. 
(a)

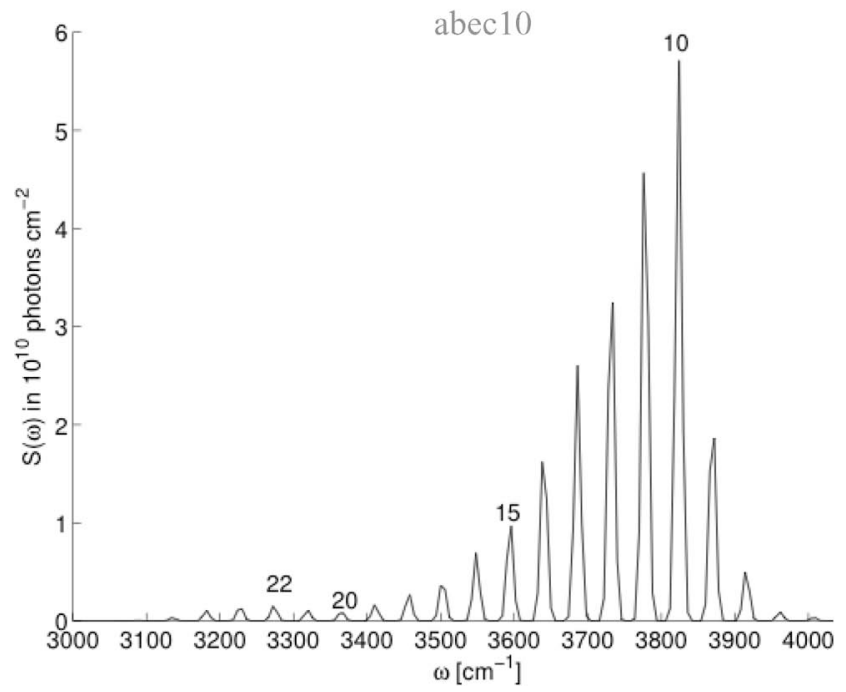

(b)

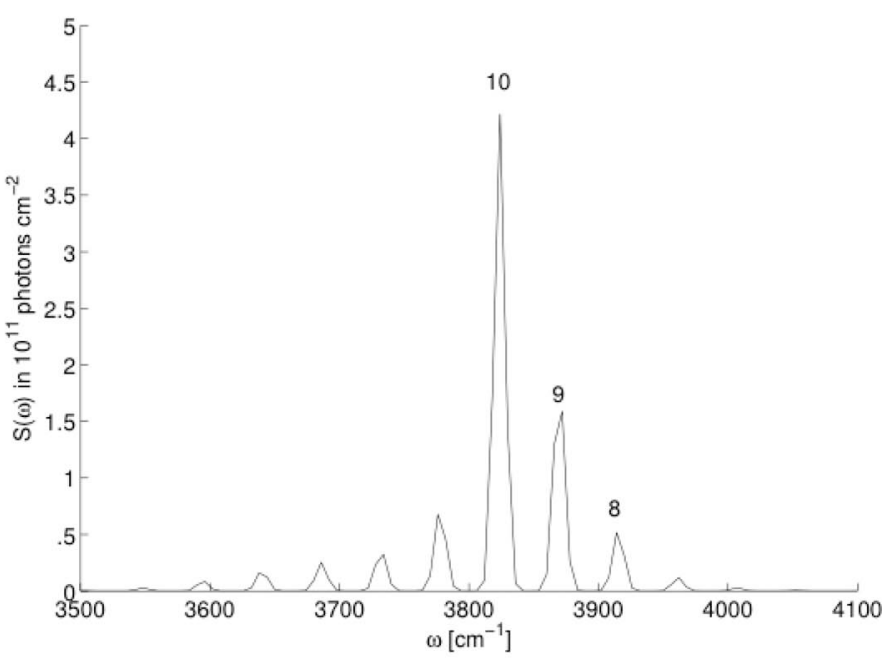

FIG. 7. The theoretical dispersed fluorescence (assuming perfect collection efficiency) after (a) $1 \mathrm{~ms}$ and (b) $8 \mathrm{~ms}$ following CLIO excitation of a monolayer (averaged over a macropulse for the highest fluence currently available at that wavelength, $k_{a b s, C L I O}=5 \times 10^{7} \mathrm{~s}^{-1}$ for $8 \mu \mathrm{s}$, or $20 \mathrm{~mJ}$ ). We note that, in (a), the appearance of a tiny higher $n$ shoulder around $n=22$ (which is even more pronounced as a relative peak at shorter times, not shown), but that the signal on the ms timescale is overwhelmed by fluorescence from lower $n$ and the distribution becomes the same as that for the monolayer under continued observation.

for monolayer fluence conditions calculated at $1 \mathrm{~ms}$, except for the significant additional computational time, as seen in Table I.

\section{DISCUSSION}

The present computational results agree with those in the previous simulation ${ }^{6,7}$ on this $\mathrm{CO} / \mathrm{NaCl}(100)$ surface in finding a vibrational pooling peak around the $n=10$ state on the ms timescale. The monolayer excitation condition in Figs. 3 and 4 can be compared directly with Figs. 3(a) and 3(b) in Ref. 7 , finding the peak around $n=10$ and similar trends on both sides of the peak.
We have described in a previous paper, ${ }^{8}$ a statistical theory of the distribution function under conditions of relatively low excitation, based on the condition imposed by one quantum assisted emission in pooling, limited by the Debye frequency, $\hbar \omega_{D}$ of the solid. The distribution function as derived in Ref. 8 is given by

$$
P_{n}(t)=\frac{m_{n}(t)}{M(t)}=\frac{e^{\gamma(t) n-\frac{\epsilon_{n}}{k T}}}{\sum_{n} e^{\gamma(t) n-\frac{\epsilon_{n}}{k T}}} .
$$

In this approximation, while in the domain of change of $n$ by energy transfer accompanied by one-phonon pooling, the $P_{n}$ are primarily constrained in the interval $[0,10]$. Clearly this (a)

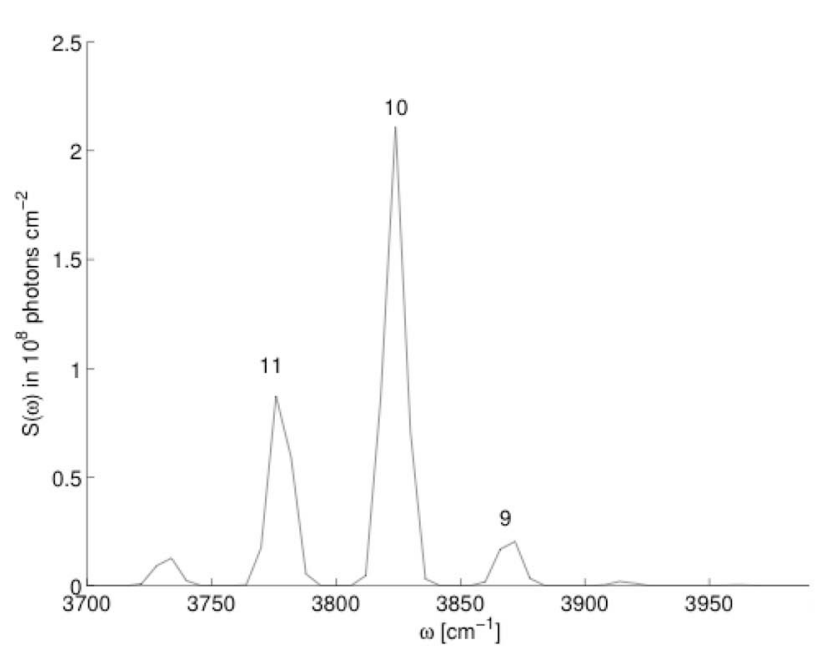

(b)

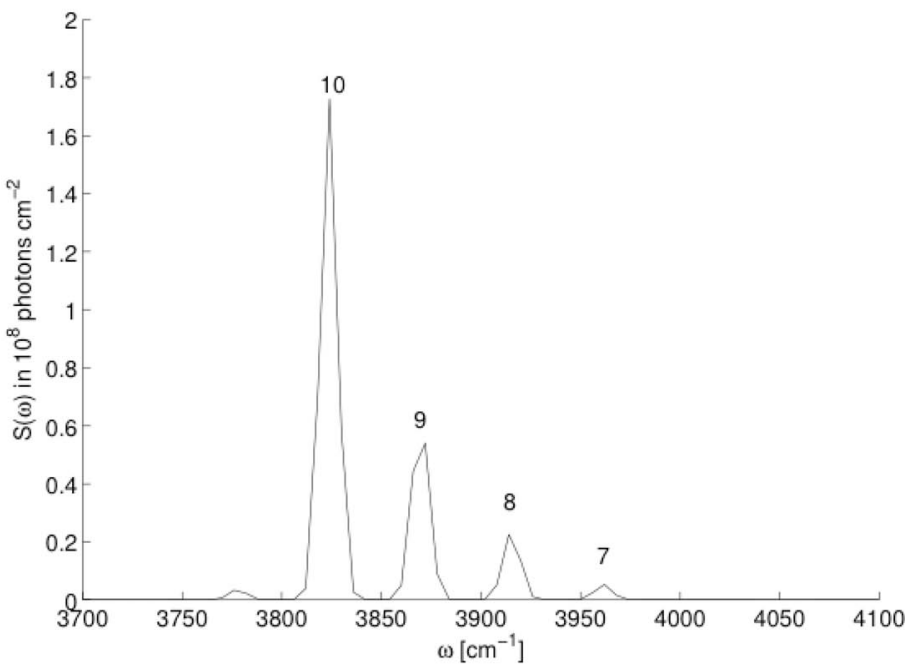

FIG. 8. Snapshots of the theoretical dispersed fluorescence for a monolayer under the experimental monolayer lasing conditions $\left(k_{a b s}=9 \times 10^{4} \mathrm{~s}^{-1}\right)$. The snapshots given are (a) $1 \mu$ s between 77 and $78 \mu$ s following beginning of excitation and (b) $12.7 \mu$ s period ending 1 ms after the beginning of lasing, representative of the difference in results at different temporal resolutions of collection. 
(a)
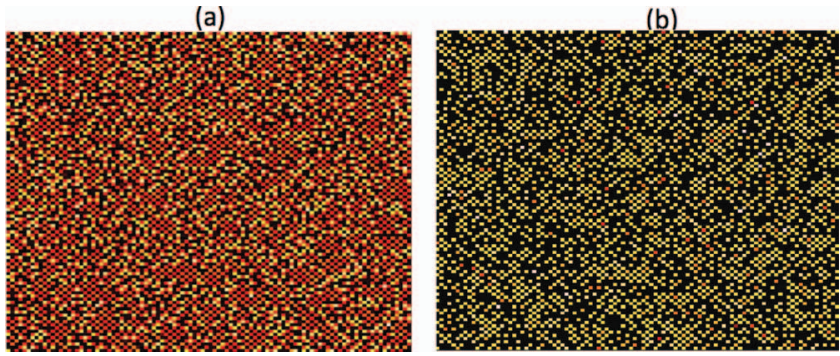

FIG. 9. The vibrational population of the surface for a single trajectory at the conclusion of a CLIO FEL pulse (figure (a), $8 \mu \mathrm{s}$ excitation) and $1 \mathrm{~ms}$ thereafter (figure (b), $k_{a b s, C L I O}=5 \times 10^{7} \mathrm{~s}^{-1}$ for $8 \mu \mathrm{s}$ excitation for both). For (a), the legend is: $\mathrm{n}=0$-black, 10-red, 20-orange, 25-yellow, 32-white, highest level. For (b) n = 0-black, 5-red, 10-yellow, 12-white, highest level.

constraint on $n$ does not hold for the CLIO excitation at short times, as in Fig. 3, but by $1 \mathrm{~ms}$ the assumption appears to hold reasonably well for both lasing conditions, as in Fig. 4. As a result, we are able to confirm in Fig. 5 that the constrained statistical theory described in a previous $\operatorname{paper}^{8}$ is in agreement with the presently calculated dependence on $n$. The systematic deviation from the Boltzmann expectation with $n$ is as theoretically expected in Eq. (10): ${ }^{8} \gamma$ is the slope of $F_{n}=\ln \left(P_{n}\right)$ $+E_{n} / k T$, vs. $n$, the vibrational state. If there is no component of the Free Energy $\left(F_{n}\right)$ which is dependent on $n$, then there should not be a dependence.

Using higher laser fluences than before and examining populations during the duration of lasing, we have found substantial populations of many $n>16$ states, as in Fig. 3, including evidence of strong inversion near the $n=22$ state $\left(3.5 \times 10^{10}\right.$ photons $\mathrm{cm}^{-2}$ after $1 \mathrm{~ms}$ of collection, as in Fig. 7). By extending earlier work to higher intensities, these high lying states have been calculated to exist in significant populations on this surface, and they result from the complex interplay of rates, some of which are given in Fig. 1. Experimentally, levels as high as the $n=26$ state were inferred to have been observed in the dispersed fluorescence of multilayers of ${ }^{13} \mathrm{C}^{18} \mathrm{O} .{ }^{3}$ The extent of the observability of higher states experimentally depends on the temporal resolution of the apparatus. To our knowledge there have been no further experiments on the $\mathrm{CO}: \mathrm{NaCl}(100)$ system since 1990, and none with a higher fluence free electron laser source of the sort described here.

As seen in Figs. 6 and 7 after $1 \mathrm{~ms}$ following excitation, the distribution is more narrowly peaked around an $n=10$ maximum at lower fluences, and becomes broader and peaked around slightly higher values at higher fluences. Over time, the evolution of pools by relaxation leads to a characteristic dispersed fluorescence signature, calculated by Eq. (9).

TABLE I. The calculation times and results for a single trajectory on a 100 $\times 100$ grid with three different resonant rate conditions $1 \mathrm{~ms}$ following monolayer excitation. They each have relative peaks at $n=10$, not shown.

\begin{tabular}{lcc}
\hline \hline Resonant rate factor & Computational time (min.) & $\mathrm{P}_{10}(1 \mathrm{~ms})$ \\
\hline $1 / 1000$ & 9 & 0.0196 \\
$1 / 100$ & 91 & 0.0206 \\
$1 / 10$ & 1179 & 0.0182 \\
\hline \hline
\end{tabular}

While the results show pronounced maxima based on the effect of the single-phonon Debye cutoff (peaks around $n=10,19$, and 27 in the figures), the results including twophonon assisted rates were indistinguishable from those including only single-phonon transfers. This is not surprising, since the two-phonon rates are expected to be significantly slower, as in Fig. 1. We have since derived a more general treatment, no longer restricted to Debye solids. It and its application will be submitted for publication.

The rate constants are given in Appendix A, but it may be instructive to recognize that the rate constant for resonant and non-resonant pooling is similar to that for Förster transfer, ${ }^{14}$ one an electronic transition dipole and the other a vibrational transition dipole. For instance, one expects the $R$ dependence of the resonant transfer rates in the present theory to be the same as Forster transfer, observable at lower surface coverages.

One can also reflect on what can be learned about the general problem of energy transfer, with the vibrational pool as an example of a dynamic bottleneck. Whether such a bottleneck results in single or stretched exponential kinetics depends on the timescale of the relaxation relative to equilibration: if the excitations last a long time relative to heat-bath-coupled transfer processes, they will reach equilibrium and single exponential kinetics will be observed. Such a conclusion is not unique to vibrational pooling, as the trapping in dye-sensitized solar cells shows such a transition from single to multiple exponential kinetics depending on the concentration of passivating $I_{2} \cdot{ }^{15}$

For all the systems with high levels of excitation, as in Fig. 9, one notices immediately a checkerboard pattern as a result of competition between different sites for the accumulation of quanta. When excitation levels are somewhat lower, as in the snapshots $1 \mathrm{~ms}$ following excitation, this competition is limited to single-phonon-assisted transfer rates, which are much faster than multi-phonon-assisted transfer. This pattern is identified as an anti-correlation between pooling peak states ( $n>1$, but typically $n=8,9$, or 10 ) or correlations between peaks and vacancies, and it occurs within $2 \mu \mathrm{s}$ after excitation, remaining anti correlated throughout all of our simulations, as long as the pool states exist (four orders of magnitude in time). As a result, the mean-field approximation of the master equation is not expected to give accurate results.

We include stimulated emission in these calculations for the first time. We suggest that the previous experiments could not measure fluorescence (instead measuring the overtone) because of the overwhelming stimulated emission signal during lasing. While arising from the much faster stimulated emission process $\left(k_{a b s}=9 \times 10^{4} \mathrm{~s}^{-1}\right)$, the photons collected would be indistinguishable from fluorescence $\left(k_{f}^{\Delta v=1}=11.4 \mathrm{~s}^{-1}\right)$. This effect also adds many more Monte Carlo steps to the calculation at the higher lasing fluence, more than doubling the computational time required.

It may appear at first unusual that, over time, the populations excited by higher intensity lasers relax to similar distributions as those at lower fluence light, since three orders of magnitude of fluence are spanned. This calculated result may be the signature of a constrained statistical behavior in a 
physically based vibrational energy distribution, as explored theoretically in a previous paper. ${ }^{8}$

\section{CONCLUSIONS}

In the present model, vibrational pooling leads to the accumulation of vibrational energy in ${ }^{13} \mathrm{CO}$ on $\mathrm{NaCl}(100)$. The model suggests substantial vibrational population inversion under existing laser conditions.

Importantly, the calculation supports the constrained vibrational population distribution recently derived theoretically for monolayers of high-frequency vibrations at solid surfaces where the assumptions of the model hold. ${ }^{8}$ Further theoretical characterization of this high fluence regime, where the previous theoretical assumptions fail, is the topic of a forthcoming work. ${ }^{16}$

The following experimentally testable predictions are also made:

1. States as high as $n=32$ may be excited with currently available laser conditions $\left(1.5 \times 10^{4}\right.$ photons $\mathrm{cm}^{-2}$ 7-8 $\mu$ s following lasing under CLIO FEL conditions), although the continued brightness of the $n \approx 10$ pooled states through the ms timescale $\left(4 \times 10^{11}\right.$ photons $\mathrm{cm}^{-2}$ after $8 \mathrm{~ms}$ as in Fig. 7(b) may restrict the observability of these higher states by dispersed fluorescence.

2. In addition to the inverted distribution with a peak at $n=10$ persisting to $20 \mathrm{~ms}$, there is predicted to be a second inverted peak in the dispersed overtone fluorescence expected to appear around $n=22,2 \times 10^{9}$ photons $\mathrm{cm}^{-2}$ after $1 \mathrm{~ms}$ of continuous collection, as in Fig. 7, when the absorbed laser fluence prior to relaxation lifetime is increased to Free Electron Laser intensity.

\section{ACKNOWLEDGMENTS}

We thank to the Office of Naval Research (ONR), U.S. Army Research Office (ARO), and National Science Foundation (NSF) for the support of this research. We also thank S. A. Corcelli for providing a copy of his programs and helpful discussions, as well as H.-C. Chang and C. Noda for their useful discussions.

\section{APPENDIX A: RATE CONSTANTS} Tully:

We use the relaxation rate expression from Corcelli and

$$
\begin{gathered}
\kappa_{n}=\sum_{p=1}^{\infty} \frac{f_{p} I_{p}}{\omega_{n} p !}|\langle n|x| n-1\rangle|^{2}\left[n\left(\frac{\omega_{n}}{p}\right)+1\right]^{p}, \\
\left.f_{p}=(-1)^{p}\left(2^{p+1}-2\right) D^{\prime} \alpha^{\prime p+1} \frac{m_{O}}{M_{C O}}\right), \\
I_{p}=\lambda^{p} \frac{\omega_{n}}{\omega_{D}} \sqrt{\frac{75 \pi}{p}} \exp \left[-\left(\frac{75}{4 p}\right)\left(\frac{\omega_{n}}{\omega_{D}}-\frac{4 p}{5}\right)^{2}\right], \\
n(\omega)=\left(e^{\frac{\omega}{k T}}-1\right)^{-1},
\end{gathered}
$$

where $p$ is the order of phonon excitation of the solid and $\lambda$ is the only free parameter (in Eq. (8)). We note two slight typos in $I_{p}$ in the original work have been identified (compared to Ref. 7), but there was no corresponding typo in the program. The remaining quantities are the temperature of the solid $(22 \mathrm{~K})$, the Debye cutoff $\omega_{D}=223 \mathrm{~cm}^{-1},{ }^{7}$ the energy levels of the CO Morse oscillator $\omega_{n}$, the mass of oxygen $m_{O}=16 \mathrm{amu}$, the total mass of $\mathrm{CO}, m_{C O}=29 \mathrm{amu}$, and the properties of the Morse coupling of the $\mathrm{CO}$ to the $\mathrm{NaCl}$, the binding energy $D^{\prime}=0.168 \mathrm{eV}$ and $\alpha^{\prime}=0.816 \AA^{-1}$.

To calculate the vibrational pooling rate constants, $W_{n, m}^{p}$ we use $e^{6,7}$

$$
\begin{aligned}
W_{m, n}^{p}= & \sum_{p=1}^{\infty} 2 \pi p ! 2^{p} g_{p}^{2}|\langle n|x| n-1\rangle|^{2} \\
& \times|\langle m|x| m+1\rangle|^{2}\left[\frac{n\left(\frac{\omega_{n, m}}{p}\right)+1}{M_{N a}}\right]^{p} H_{p}, \\
g_{p}=(-1)^{p} \frac{\mu^{\prime 2}(p+1)(p+2)}{2 R_{0}^{p+3}} & \\
H_{p}= & \int_{0}^{\infty} \mathrm{d} \omega_{1} \cdots \int_{0}^{\infty} \mathrm{d} \omega_{p} \frac{\rho\left(\omega_{1}\right)}{\omega_{1}} \cdots \frac{\rho\left(\omega_{p}\right)}{\omega_{p}} \\
& \times \delta\left(\omega_{m, n}-\omega_{1}-\cdots-\omega_{p}\right),
\end{aligned}
$$

noting that depooling rates $W_{m, n}^{p}=W_{m+1, n-1}^{d} e^{\beta \omega_{n, m}}$ are also known immediately by detailed balance. Besides $p$, the number of phonons of the solid needed to mediate the transfer, the other parameters are: the energy dissipated by the pooling $\omega_{m, n}=\omega_{n}-\omega_{m+1}$, the site-to-site distance between nearest neighbor CO molecules $R_{0}=3.96 \AA$, the mass of the $\mathrm{Na}^{+}$ion $M_{N a}=23 \mathrm{amu}$, and the normalized phonon density of states $\rho=3 \omega^{2} / \omega_{D}^{3}$ for $\omega<\omega_{D}=223 \mathrm{~cm}^{-1}$, for a Debye solid. ${ }^{6,7}$

The $k_{n}^{\Delta n=i}$, fluorescence rate constant for a $\mathrm{CO}$ with $n$ vibrational energy quanta, are obtained from the standard relations: ${ }^{17}$

$$
\begin{aligned}
k_{n}^{\Delta n=1} & =\frac{16 \pi^{3} \omega_{n}^{3} \mu^{\prime 2}}{3 \epsilon_{0} h c^{3}}|\langle n|x| n-1\rangle|^{2}, \\
k_{n}^{\Delta n=2} & =\frac{16 \pi^{3} \omega_{n \Delta 2}^{3} \mu^{\prime 2}}{3 \epsilon_{0} h c^{3}}|\langle n|x| n-2\rangle|^{2},
\end{aligned}
$$

where $\omega_{n \Delta 2}$ is the frequency of the $n$ to $n$ - 2 overtone fluorescence, $\mu^{\prime}$ is the coefficient in the transition dipole moment, $d \mu(x) /\left.d x\right|_{x=0}\left(1.8 \mathrm{D} / \AA\right.$ in the case of $\left.{ }^{13} \mathrm{CO}\right),{ }^{7}$ and the matrix elements can be evaluated, for example, in a Morse oscillator basis. Following Gallas, ${ }^{18}$ the dipole operator matrix elements for the Morse oscillator eigenstates are given by

$$
\begin{gathered}
\langle n|x| n-1\rangle=\frac{1}{a(k-2 n)}\left(\frac{n(k-2 n-1)(k-2 n+1)}{(k-n)}\right)^{1 / 2}, \quad \text { (A10) } \\
\langle n|x| n-2\rangle=\frac{-1}{2 a(k-2 n+1)}\left(\frac{n(n-1)(k-2 n-1)(k-2 n+3)}{(k-n+1)(k-n)}\right)^{1 / 2},
\end{gathered}
$$

where the Morse constants are $a=2.209 \AA^{-1}$ and $D=12.3 \mathrm{eV}$ and $k=\omega_{e} / \omega_{e} \chi_{e}=185, \omega_{e}=2130 \mathrm{~cm}^{-1}$ is the fundamental frequency of the Morse oscillator and $\omega_{e} \chi_{e}=11.50 \mathrm{~cm}^{-1}$ is the anharmonicity (such that 
$\left.\omega_{2}=2084 \mathrm{~cm}^{-1}\right)$. The resulting overtone fluorescence rate constants range from $k_{2}^{\Delta n=2}=0.239 \mathrm{~s}^{-1}$ to $k_{44}^{\Delta n=2}=60.2 \mathrm{~s}^{-1}$. The fundamental fluorescence rate constants range from $k_{1}^{\Delta n=1}=11.6 \mathrm{~s}^{-1}$ to $k_{26}^{\Delta n=1}=135 \mathrm{~s}^{-1}$.

\section{APPENDIX B: UNITS}

To connect with units traditionally used in chemical kinetics, we describe the population evolution equations below, adapting $\left(d P_{n} / d t\right)_{p d r f}$ in Eq. (5) to more familiar units. Let $M_{n}$ be the number of sites that have $n$ quanta. We then have $P_{n}=M_{n} / M$. Let $P(m \mid n)$ be the conditional probability, in particular the probability that given a site with $n$ quanta, there is an adjacent site with $m$ quanta. So in Eq. (5) we replace the $P_{n, m}$ 's with $P_{n} P(m \mid n)$ 's. The $P_{n}$ 's replace the normal concentrations $c_{n}$ in kinetics,

$$
\begin{aligned}
&{\frac{d P_{n}}{d t}{ }_{p d}=} \sum_{m}\left(W_{n-1, m}^{p}\right) P_{n-1} P(m \mid n-1) \\
&+\sum_{m}\left(W_{n+1, m}^{d}\right) P(m \mid n+1) P_{n+1} \\
&-\sum_{m}\left(W_{n, m}^{p}\right) P(m \mid n) P_{n}-\sum_{m}\left(W_{n, m}^{d}\right) P(m \mid n) P_{n} .
\end{aligned}
$$

As a result, the units of the second-order rates are technically per conditional probability of an $m$ neighbor, given an $n$-site under consideration.

${ }^{1}$ H. C. Chang, D. J. Dai, and G. E. Ewing, J. Chin. Chem. Soc. 42, 317 (1995).

${ }^{2}$ H.-C. Chang, H. H. Richardson, and G. E. Ewing, J. Chem. Phys. 89, 7561 (1988).

${ }^{3}$ H.-C. Chang and G. E. Ewing, J. Phys. Chem. 94, 7635 (1990).

${ }^{4}$ H.-C. Chang, C. Noda, and G. E. Ewing, J. Vac. Sci. Technol. 8, 2644 (1990).

${ }^{5}$ H.-C. Chang and G. E. Ewing, Phys. Rev. Lett. 65, 2125 (1990).

${ }^{6}$ S. A. Corcelli and J. C. Tully, J. Phys. Chem. A 106, 10849 (2002).

${ }^{7}$ S. A. Corcelli and J. C. Tully, J. Chem. Phys. 116, 8079 (2002).

${ }^{8}$ E. T. D. Boney and R. A. Marcus, J. Chem. Phys. 139, 124107 (2013).

${ }^{9}$ H.-C. Chang and G. E. Ewing, Chem. Phys. 139, 55 (1989).

${ }^{10}$ K. A. Fichthorn and W. H. Weinberg, J. Chem. Phys. 95, 1090 (1991).

${ }^{11}$ L. MacAleese, A. Simon, T. B. McMahon, J.-M. Ortega, D. Scuderi, J. Lemaire, and P. Maître, Int. J. Mass. Spectrom. 249, 14 (2006).

${ }^{12}$ D. S. Anex and G. E. Ewing, J. Phys. Chem. 90, 1604 (1986).

${ }^{13}$ H.-C. Chang, private communication (2011).

${ }^{14}$ T. Förster, Discuss. Faraday Soc. 27, 7 (1959).

${ }^{15}$ A. N. M. Green, R. E. Chandler, S. A. Haque, J. Nelson, and J. R. Durrant, J. Phys. Chem. B 109, 142 (2005).

${ }^{16}$ E. T. D. Boney and R. A. Marcus, "Vibrational pooling: Chemical potential and correlation," J. Chem. Phys. (unpublished).

${ }^{17}$ P. F. Bernath, Spectra of Atoms and Molecules (Oxford University Press, NY, 2005).

${ }^{18}$ J. A. C. Gallas, Phys. Rev. A 21, 1829 (1980). 